\title{
Research on the output bit error rate of 2 DPSK signal based on stochastic resonance theory
}

\author{
Daqin Yan*, Fuzhong Wang ${ }^{\dagger}$ and Shuo Wang ${ }^{\ddagger}$ \\ School of Science, Tianjin Polytechnic University, \\ Tianjin 300387, China \\ *13502086683@163.com \\ †wangfuzhong@163.com \\ ‡cc611108@163.com
}

Received 14 May 2017

Revised 17 July 2017

Accepted 30 November 2017

Published 8 December 2017

\begin{abstract}
Binary differential phase-shift keying (2DPSK) signal is mainly used for high speed data transmission. However, the bit error rate of digital signal receiver is high in the case of wicked channel environment. In view of this situation, a novel method based on stochastic resonance (SR) is proposed, which is aimed to reduce the bit error rate of 2DPSK signal by coherent demodulation receiving. According to the theory of SR, a nonlinear receiver model is established, which is used to receive 2DPSK signal under small signalto-noise ratio (SNR) circumstances (between $-15 \mathrm{~dB}$ and $5 \mathrm{~dB}$ ), and compared with the conventional demodulation method. The experimental results demonstrate that when the input SNR is in the range of $-15 \mathrm{~dB}$ to $5 \mathrm{~dB}$, the output bit error rate of nonlinear system model based on SR has a significant decline compared to the conventional model. It could reduce $86.15 \%$ when the input SNR equals $-7 \mathrm{~dB}$. Meanwhile, the peak value of the output signal spectrum is 4.25 times as that of the conventional model. Consequently, the output signal of the system is more likely to be detected and the accuracy can be greatly improved.
\end{abstract}

Keywords: Stochastic resonance; 2DPSK signal; bit error rate; nonlinear bistable system; signal-to-noise ratio.

\section{Introduction}

Binary differential phase-shift keying (2DPSK) transmits the digital information using carrier wave's relative phase change of adjacent code element, also called binary relative phase-shift keying (PSK). 2DPSK has advantages over amplitude-shift keying (ASK) and frequency-shift keying (FSK) in terms of the anti-noise performance

${ }^{\dagger}$ Corresponding author.

This is an Open Access article published by World Scientific Publishing Company. It is distributed under the terms of the Creative Commons Attribution 4.0 (CC-BY) License. Further distribution of this work is permitted, provided the original work is properly cited. 
and the channel frequency band utility. At the same time, 2DPSK is not easily influenced by the change of channel characteristics. 2DPSK overcomes the problem of carrier phase ambiguity ${ }^{1}$ in coherent demodulation by PSK. 2DPSK is a kind of easy and practical digital phase modulation system, which has been widely applied in the high speed data transmission. The conventional demodulation methods of 2DPSK signal contain coherent demodulation (polarity comparison method) added code inverse transform and differential coherent demodulation (phase comparison method). ${ }^{2}$ While these methods require high signal-to-noise ratio (SNR), they are unable to demodulate weak 2DPSK signal.

In 1981, Italy scholar Benzi et al. first put forward the concept of stochastic resonance $(\mathrm{SR})^{3}$ in the research of Ice Periodic, which is brought up to address the phenomenon of the freezing period and the heating period occurring alternatively about every 100,000 years in the ancient weather. Different from that, the noise is used as the interference term in the traditional method, SR is in the use of noise to enhance the energy of the weak signal under the synergistic effect of the input signal and the noise in the nonlinear system, so as to achieve the purpose of identifying the weak signals. SR has continuously attracted the considerable attention from various fields and it has been applied in machine tactile,,$\frac{4}{,}$ image processing,,$\frac{5}{4}$ electronic circuit, $\frac{6}{-}$ signal detection, $\frac{7}{-}$ etc. However, most of the applications of SR are in analog signal processing. The researches on the applications of SR in digital signal processing are relatively few.

This paper presents a new method for the demodulation of weak 2DPSK signals based on the theory of SR. Nonlinear demodulation method based on the theory of $\mathrm{SR}$ is analyzed and compared with the conventional demodulation method in the condition of the heavy background noise with the SNR less than $0 \mathrm{~dB}$ from three cases that contain the time-domain, the frequency-domain and the bit error rate of the system output. The advantage of SR in the digital signal processing can be found.

\section{The Establishment of 2DPSK Signal SR System Model and Its Principle}

SR system model satisfies the Langevin equation ${ }^{8-10}$ :

$$
d x / d t=a x(t)-b x^{3}(t)+s(t)+n(t),
$$

where $a$ and $b$ denote the nonlinear bistable coefficients with positive real value; $n(t)$ represents the additive Gaussian white noise with the zero mean and intensity $D$; the input signal $s(t)$ is the 2DPSK signal; $x(t)$ is the output signal; this system can be regarded as a nonlinear signal receiver. Assume that $g(t)$ is the absolute code of transmittal digital code element and $k(t)$ is the relative code. According to the principle of differential encoding: $k(t)=g(t) \oplus k\left(t-T_{s}\right), k(t)$ is obtained by $g(t)$ based on differential encoding. Then the 2DPSK signal can be obtained by $k(t)$ through modulating, whose impression in the duration of an element, namely 
$T_{s}$, is given as

$$
s(t)=A \cos \left[w_{c} t+k(t) \pi\right], \quad k(t)=1 \text { or } 0 .
$$

$s(t)$ can also be described as

$$
s(t)=\left\{\begin{array}{ll}
u_{1 T}(t), & k(t)=0 \\
u_{0 T}(t)=-u_{1 T}(t), & k(t)=1
\end{array},\right.
$$

in which

$$
u_{1 T}(t)=\left\{\begin{array}{ll}
A \cos w_{c} t, & 0<t<T_{s} \\
0, & \text { others }
\end{array} .\right.
$$

When there is no signal and noise into the system, at this time, $A=0, D=0$. The potential function ${ }^{11}$ of the nonlinear bistable system is expressed as

$$
V(x)=-a x^{2} / 2+b x^{4} / 4 .
$$

The two potential wells and one potential barrier at $x= \pm \sqrt{a / b}$ and $x=0$ are, respectively, corresponding to the two minimum points and one maximum point of the potential function curve. When $A \neq 0, D=0$, the critical value of the input signal is $A_{c}=\sqrt{4 a^{3} / 27 b}$. Meanwhile, if the amplitude of the input signal meets $A<A_{c}$, the particle can only do local periodic motion with the same frequency in one of the two potential wells. When $A \neq 0, D \neq 0$, although $A<A_{c}$, the particle can obtain energy from the noise through the nonlinear bistable system, jumping from the original well into another potential well, which makes the system output signal.

The conventional coherent demodulation (polarity comparison method) added code inverse transform system model and the SR nonlinear system model of 2DPSK signal are shown, respectively, in Figs. 1(a) and 1(b).

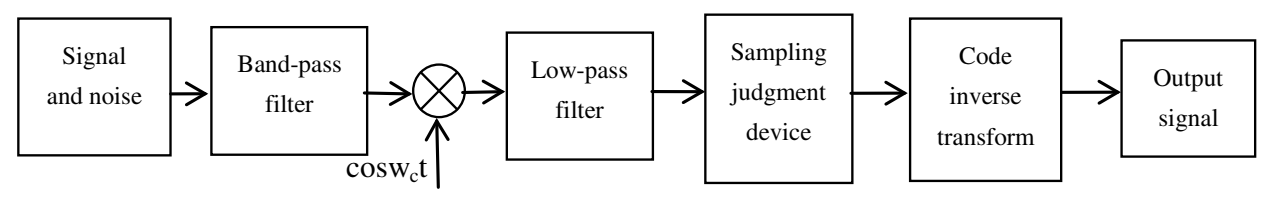

(a) The coherent demodulation added code inverse transform system model

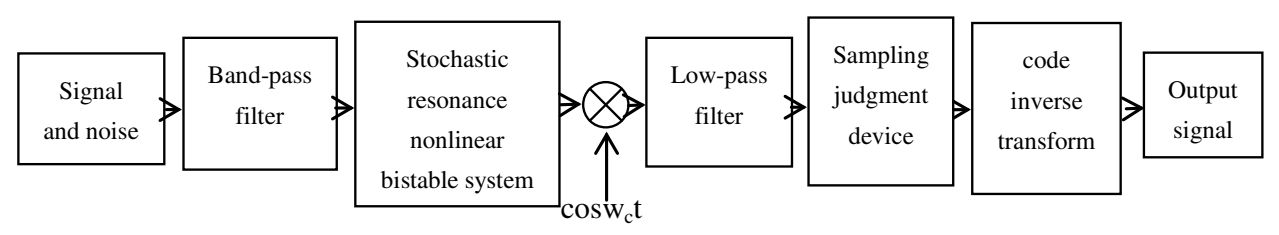

(b) The stochastic resonance nonlinear system model

Fig. 1. 2DPSK signal receiving model. 


\section{Research on the Theory Bit Error Rate of 2DPSK Signal SR System Output}

The simplified model of 2DPSK signal SR coherent demodulation system model, namely Fig. 1(b), is shown in Fig. 2.

Here, $P_{e}^{\prime}$ is the bit error rate of 2 DPSK signal through nonlinear system when adopting polarity comparison-code inverse transform method. $P_{e}$ is the bit error rate of 2PSK signal through the nonlinear system when adopting coherent demodulation, that is, the bit error rate of the input port of the code inverse transform device. Therefore, $P_{e}^{\prime}$ needs to consider only the influence of code inverse transform device on the bit error rate based on $P_{e}$.

$P_{e}$ is discussed. Assume that the sampling time of the channel output signal is $\Delta t$, and keeping one-order hold. Within $\Delta t$, the signal and the noise can be described as

$$
s_{i}(n \Delta t)+n_{i}(n \Delta t)
$$

in which $i=1,2, \ldots, n=1,2, \ldots$ Substitute Eq. (6) into Eq. (1), so

$$
\begin{aligned}
d x / d t= & a x(t)-b x^{3}(t)+s_{i}(n \Delta t)+n_{i}(n \Delta t), \\
& (n-1) \Delta t \leq t<n \Delta t, \quad n=1,2, \ldots .
\end{aligned}
$$

After the nonlinear system, the output of the system is still a random process, using the mean of the output as the output signal. In a symbol interval, the sampling value of the system output signal is constant through the coherent demodulation, set as $S$, then $S= \pm A$. Plugging $S$ into Eq. (7), then,

$$
d x / d t=a x(t)-b x^{3}(t)+S+n_{i}(n \Delta t) .
$$

Transform Eq. (8) as follows: $y=x / \sqrt{D}, \bar{b}=b D, \bar{S}=S / \sqrt{D}$. Then, Eq. (8) can be expanded as

$$
d y / d t=a y-\bar{b} y^{3}+\bar{S}+\xi(t),
$$

where $\xi(t)$ is the normalized noise with $\langle\xi(t) \xi(0)\rangle=2 \delta(t)$, and the SR phenomenon can be achieved by adjusting the parameters of the system. The output probability density function $\rho(y, t)$ of 2PSK signal nonlinear model represented by Eq. (9) satisfies the Fokker-Planck equation ${ }^{12}$ :

$$
\partial \rho(y, t) / \partial t=-\partial[\bar{C}(y) \rho(y, t)] / \partial y+\partial^{2} \rho(y, t) / \partial y^{2},
$$

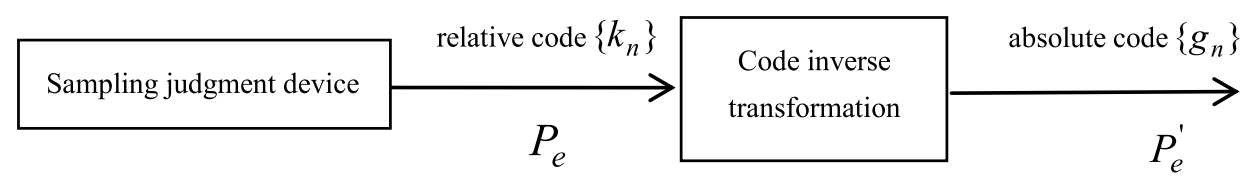

Fig. 2. The simplified model of 2DPSK signal receiving model. 
in which $\bar{C}(y)=a y-\bar{b} y^{3}+\bar{S}$. The asymptotic solution of this equation ${ }^{13}$ is expressed as

$$
\rho(y)=\lim _{t \rightarrow+\infty} \rho(y, t)=C_{0} \exp [g(y)],
$$

where $g(y)=1 / 2 a y^{2}-1 / 4 \bar{b} y^{4}+\bar{S} y, C_{0}$ is obtained by the normalization condition of probability density. According to the symmetry of probability density function distribution, the optimal judgment threshold value is 0 . At this moment, the wrong judgment probability of sending " 1 " to " 0 " is described as

$$
p(0 / 1)=\int_{-\infty}^{0} \rho\left(y \mid+A_{m}\right) d y .
$$

The wrong judgment probability of sending " 0 " to " 1 " is described as

$$
p(1 / 0)=\int_{0}^{+\infty} \rho\left(y \mid-A_{m}\right) d y,
$$

in which $A_{m}=\bar{S}=A / \sqrt{D}$. In conclusion, the total error rate of 2PSK signal through nonlinear coherent demodulation system is presented as

$$
p_{e}=p(0) p(1 / 0)+p(1) p(0 / 1) \text {. }
$$

The influence of the code inverse transformation on the error code is analyzed. Assume that the relative code sequence is $\left\{k_{n}\right\}$ and the absolute code sequence of the code inverse converter output is $\left\{g_{n}\right\}$. The condition of wrong code in $\left\{g_{n}\right\}$ is considered when there is one error code, two continuous error codes, $\ldots, n$ continuous error codes in $\left\{k_{n}\right\}$. According to the principle of the differential decoding (code inversion): $g_{n}=k_{n} \oplus k_{n-1}$, one error code in $\left\{k_{n}\right\}$ through the code inverse converter makes $\left\{g_{n}\right\}$ generated two error codes; if there are two continuous error codes in $\left\{k_{n}\right\},\left\{g_{n}\right\}$ has only two error codes; even though there are $n(n>2)$ continuous error codes in $\left\{k_{n}\right\},\left\{g_{n}\right\}$ has only two error codes located at both the ends. Assuming that the error probability of each code is equal and statistically independent, $P_{e}^{\prime}$ can be described as

$$
P_{e}^{\prime}=2 P_{1}+2 P_{2}+\cdots+2 P_{n}+\cdots
$$

where $P_{n}$ is the probability of $n$ continuous error codes in $\left\{k_{n}\right\}$. Further analysis is conducted as

$$
\begin{aligned}
P_{1} & =\left(1-P_{e}\right) P_{e}\left(1-P_{e}\right)=\left(1-P_{e}\right)^{2} P_{e}, \\
P_{2} & =\left(1-P_{e}\right) P_{e}^{2}\left(1-P_{e}\right)=\left(1-P_{e}\right)^{2} P_{e}^{2}, \\
& \vdots \\
P_{n} & =\left(1-P_{e}\right) P_{e}^{n}\left(1-P_{e}\right)=\left(1-P_{e}\right)^{2} P_{e}^{n} .
\end{aligned}
$$

On substituting Eq. (16) into Eq. (15), $P_{e}^{\prime}$ can be obtained as

$$
\begin{aligned}
P_{e}^{\prime} & =2\left(1-P_{e}\right)^{2}\left(P_{e}+P_{e}^{2}+\cdots+P_{e}^{n}+\cdots\right) \\
& =2\left(1-P_{e}\right)^{2} P_{e}\left(1+P_{e}+P_{e}^{2}+\cdots+P_{e}^{n}+\cdots\right) .
\end{aligned}
$$


Because $P_{e}$ is less than 1 , the following formulation must be correct:

$$
\left(1+P_{e}+P_{e}^{2}+\cdots+P_{e}^{n}+\cdots\right)=\frac{1}{1-P_{e}} .
$$

On substituting Eq. (18) into Eq. (17), $P_{e}^{\prime}$ can be further expressed as

$$
\begin{aligned}
P_{e}^{\prime} & =2\left(1-P_{e}\right) P_{e} \\
& =2\{1-[p(0) p(1 / 0)+p(1) p(0 / 1)]\}[p(0) p(1 / 0)+p(1) p(0 / 1)] .
\end{aligned}
$$

\section{The Numerical Experiment}

\subsection{The analysis of the output signal in the time-domain and the frequency-domain}

The 2DPSK signal with the amplitude $A=0.3$ and the input $\mathrm{SNR}=-8 \mathrm{~dB}$ is coherently demodulated by the models in Figs. 1(a) and 1(b). The parameters $a$

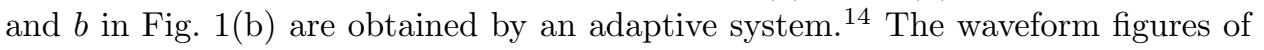
numerical experiments are as follows.

The system responses in the time-domain, respectively, output by the two models are shown in Fig. 3. It can be seen from Fig. 3(a) that the output signal is seriously interfered by the noise through the traditional model, resulting in that the signal waveform is completely submerged. The output signal waveform through the SR model in Fig. 3(b) is relatively clear, approximately periodic signal. It indicates that the SR phenomenon occurs when 2DPSK signal and the noise are via SR nonlinear bistable system. According to the theory of SR, the partial noise energy is transformed into the signal energy when the signal and the noise pass through the SR system, which weakens the interference with the signal from the noise.

The system response spectra, respectively, output by the two models are shown in Fig. 4. The carrier frequency's amplitude of 2DPSK signal is 19,094 in Fig. 4(a), while 81,160 in Fig. 4(b). A comparison of Fig. 4(a) with Fig. 4(b) is made, which shows that the peak value of the output spectrum through the $\overline{\mathrm{SR}}$ nonlinear system model is 4.25 times as large as through the traditional model. It verifies that the carrier frequency can be more accurately detected by using SR nonlinear coherent demodulation method compared with the traditional linear coherent demodulation method.

\subsection{The analysis of the bit error rate}

The numerical experiments of the two kinds of coherent demodulation models are conducted by the MATLAB. For 2DPSK signal, the frequency $\left(f_{c}\right)$ of the carrier signal is $0.01 \mathrm{~Hz}$, the element cycle $(T)$ of the binary base band signal is $100 \mathrm{~s}$, the code number $(N)$ of the numerical experiment is 500 . The input SNR varies from $-15 \mathrm{~dB}$ to $5 \mathrm{~dB}$. Because the signal will produce a certain degree of attenuation 


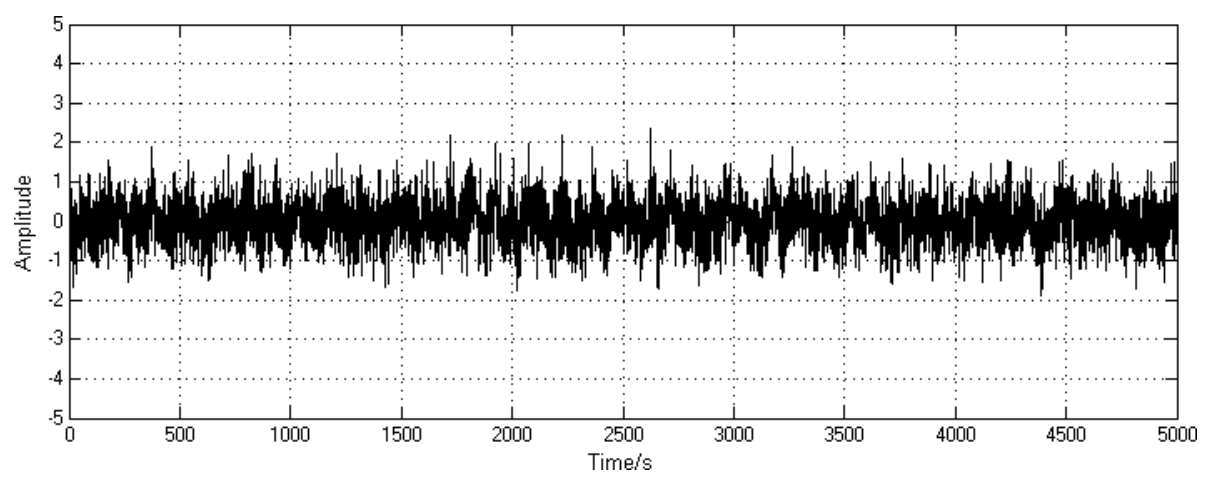

(a) Waveform in the time-domain through Fig. 1(a) model

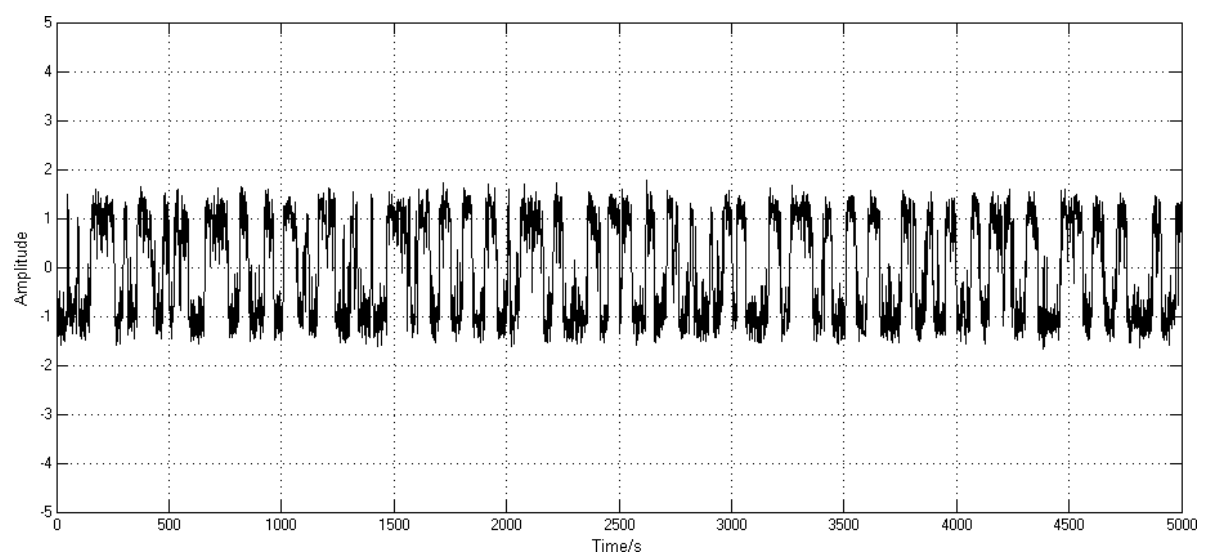

(b) Waveform in the time-domain through Fig. 1(b) model

Fig. 3. The time-domain waveform of 2DPSK signal through models in Figs. 1(a) and 1(b).

through the channel, the amplitude of 2DPSK modulating signal is in the use of $0.3,0.4,0.5$ and 0.6 , less than 1 .

The curves of the output bit error rate are shown in Fig. 5, respectively, through the SR nonlinear system model and the traditional linear model. It can be seen from the graph that the traditional linear model has a large degree of instability, while the SR nonlinear system model is relatively stable. When the input SNR is less than $3 \mathrm{~dB}$, the bit error rate curve of the SR nonlinear system model is lower than the bit error rate curve of the traditional model. When the input SNR is between $3 \mathrm{~dB}$ and $5 \mathrm{~dB}$, the bit error rate of the two models is close.

In Fig. 5(a), the output bit error rate of the SR nonlinear system model has a certain degree of decline compared to the traditional model when the input SNR is between $-15 \mathrm{~dB}$ and $-14 \mathrm{~dB}$. When the input SNR is between $-14 \mathrm{~dB}$ and $-5.5 \mathrm{~dB}$, the bit error rate curve of the SR nonlinear system model decreases rapidly, whose absolute value of the curve slope $(K=0.049)$ is significantly larger than the absolute value of the curve slope $(K=0.0249)$ of the traditional model. 


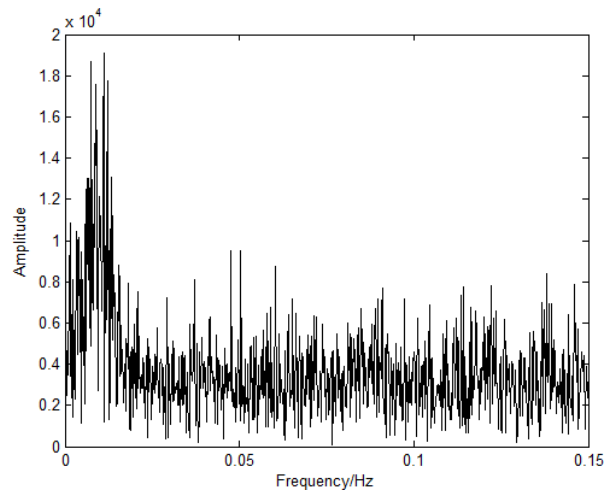

(a) Frequency spectrum through Fig. 1(a) model

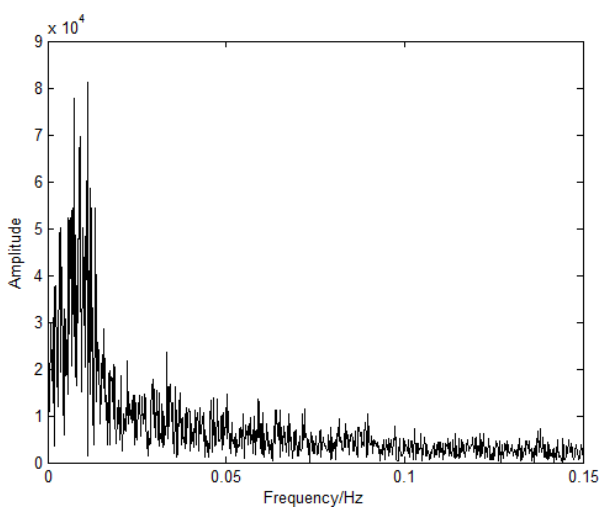

(b) Frequency spectrum through Fig. 1(b) model

Fig. 4. Frequency spectrum of 2DPSK signal through models in Figs. 1(a) and 1(b).

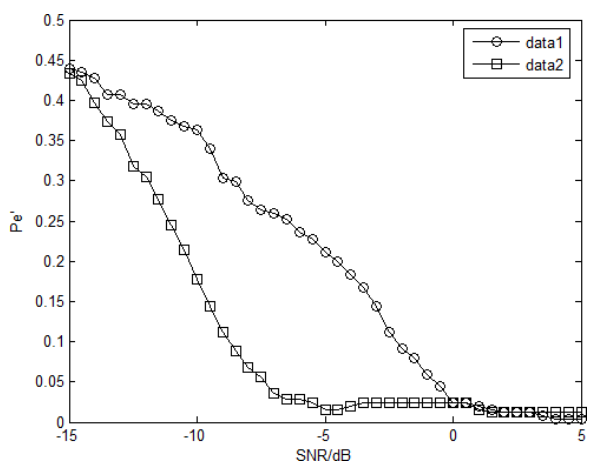

(a) $A=0.3, N=500$

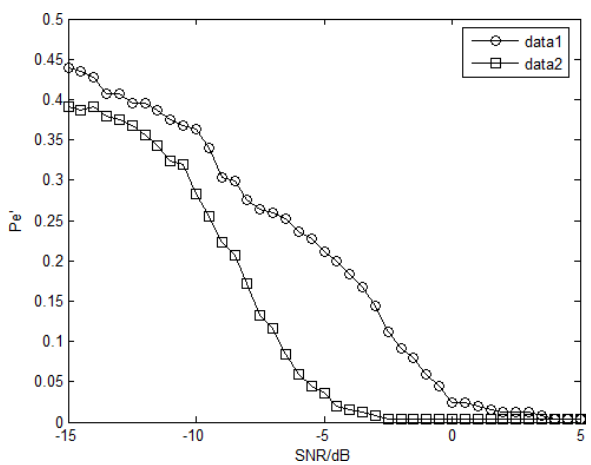

(c) $A=0.5, N=500$

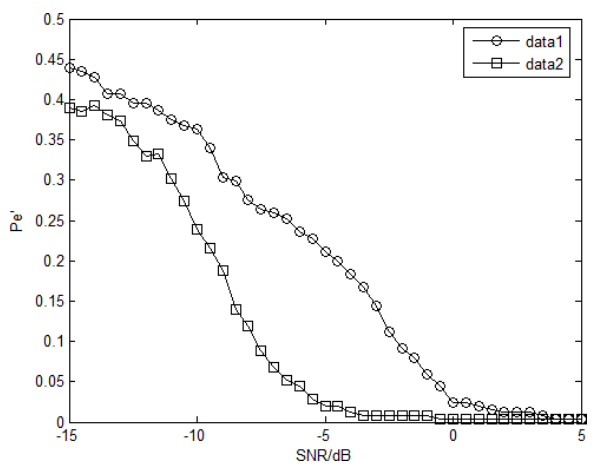

(b) $A=0.4, N=500$

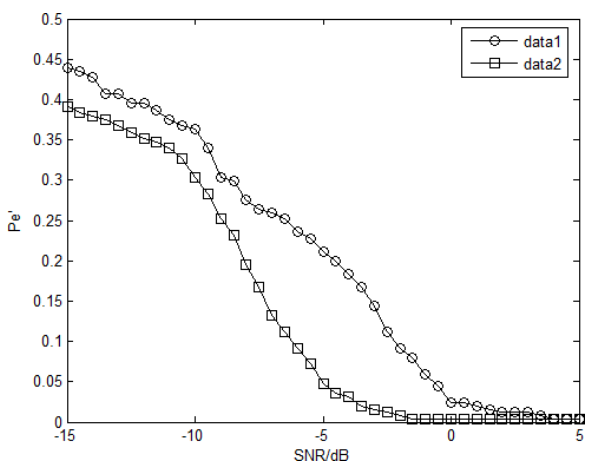

(d) $A=0.6, N=500$

Fig. 5. The comparison of the output bit error rate between the SR nonlinear system model and the conventional linear model. (The data1 is the bit error rate of 2DPSK signal by the traditional linear demodulation, while the data 2 is the bit error rate of 2 DPSK signal by the nonlinear receiver.) 
For example, the bit error rate can decrease by $86.15 \%$ in $-7 \mathrm{~dB}$. Therefore, it is shown that the accuracy of the information obtained by using the SR method is higher than that of the traditional method when receiving 2DPSK signal under heavy background noise. When the input SNR is between $-5.5 \mathrm{~dB}$ and $5 \mathrm{~dB}$, the bit error rate curve of the SR nonlinear system model changes smoothly. It can be seen from the figures that adding an SR nonlinear system at the receiver can effectively reduce the bit error rate of the system output when the input SNR is less than $0 \mathrm{~dB}$. Figures $5(\mathrm{~b})-5(\mathrm{~d})$ are the bit error rate curves of the system output obtained by the numerical experiments under the different amplitudes of the input signal compared with Fig. 5(a). It can be seen from Fig. 5 that the trend of the bit error rate curve based on SR nonlinear system model is almost the same, which shows that the SR nonlinear system model in Fig. 1(b) is stable.

The comparison of the output bit error rate of the four different signal amplitudes, namely, $A=0.3, A=0.4, A=0.5$ and $A=0.6$, is shown in Fig. 6. According to Fig. 6 , when the input SNR is from $-13.5 \mathrm{~dB}$ to $-4 \mathrm{~dB}$ and at the same SNR, the smaller the amplitude is, the lower the bit error rate is. When the periodic signal and the noise input the system at the same time, SR occurs under the interaction in the nonlinear system itself, the periodic signal and the noise, which causes that the system output signal is a kind of periodic vibration in the same frequency with the input cycle signal. In this process, part of the noise energy is converted into the signal energy. When the input SNR is same, the smaller the signal amplitude is, the more noise energy is converted into the signal energy when the SR occurs, the more obvious the phenomenon of SR is, the lower the output bit error rate is.

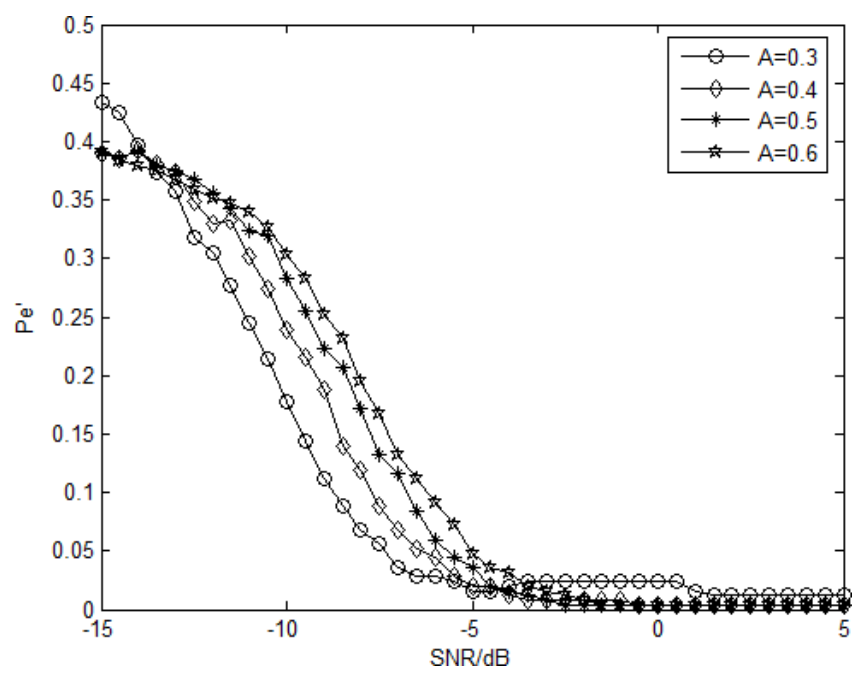

Fig. 6. The curve of the output bit error rate under the different signal amplitude $\left(f_{c}=0.01 \mathrm{~Hz}\right.$, $N=500)$. 


\section{Conclusion}

In this paper, the nonlinear receiver model is established on the basis of the principle of SR, the SR phenomenon of 2DPSK signal through nonlinear bistable system is studied. When the input SNR is between $-15 \mathrm{~dB}$ and $5 \mathrm{~dB}$, the SR system model is superior to the conventional model found in three aspects, namely, the time-domain, the frequency-domain and the system output bit error rate, by the comparison of 2DPSK signal, respectively, through the SR nonlinear system model and the conventional linear coherent demodulation model. The experimental results indicate that when adding a nonlinear receiver at the receiving end, the output time-domain waveform of 2DPSK signal has obvious periodicity compared to the traditional model. At the same time, the peak value of the output signal spectrum is 4.25 times as that of the traditional model. The results show that the carrier signal frequency can be more accurately detected by using the method of the SR nonlinear system. In addition, when the input SNR is in the range of $-15 \mathrm{~dB}$ to $5 \mathrm{~dB}$, the output bit error rate has dropped to a large extent compared to that of the traditional model, whose absolute value of curve slope $(K=0.049)$ is significantly higher than the absolute value of the curve slope $(K=0.0249)$ of the traditional model. For instance, the bit error rate could decrease by $86.15 \%$ in $-7 \mathrm{~dB}$. It is shown that the accuracy is higher when receiving 2DPSK signal in the use of the SR method under the background of strong noise.

\section{References}

1. X. Wang, W. X. Liu and G. F. Sun, J. Navigation 69 (2016) 1393.

2. F. J. Zhang, L. Y. Sheng and Y. Y. Liu, Microelectronics 43 (2013) 37.

3. R. Benzi, A. Sutera and A. Vulpiani, J. Phys. A 14 (1981) 453.

4. M. B. Ignacio et al., J. Neurophysiol. 114 (2015) 2132.

5. V. P. Subramanyam and P. K. Roy, Magn. Reson. Imaging 28 (2010) 1361.

6. A. A. Zaikin, K. Murali and J. Kurths, Phys. Rev. E 63 (2001) 45.

7. J. K. Douglass, L. Wilkens and E. Pantazelou, Nature 365 (1993) 337.

8. T. Yu, L. Zhang and M. K. Luo, Phys. Scripta 88 (2013) 5008.

9. G. Q. Zhu, K. Ding and Y. Zhang, Acta Phys. Sin. 59 (2010) 3001.

10. Y. F. Zhan and C. W. Duan, The application of stochastic resonance in parameter estimation for PSK signals, in IEEE Int. Conf. Communication Software and Networks (ICCSN) (2015).

11. R. Kihara, Y. Yamanashi and N. Yoshikawa, IEEE T. Appl. Supercon. 26 (2016) 574.

12. H. Risken, The Fokker-Planck Equation (Springer, New York, 1983).

13. X. L. Han, W. T. Lin and Y. H. Xu, Acta Phys. Sin. 63 (2014) 764.

14. J. Wang, Q. B. He and F. R. Kong, IEEE T. Instrum. Meas. 64 (2015) 564. 